\title{
A scoring system to predict the risk of postoperative complications after laparoscopic liver resection in elderly hepatocellular carcinoma patients
}

\author{
Boram LEE, Jai Young CHO*, Ho-Seong HAN, Yoo-Seok YOON, Hae Won LEE, Jun Suh LEE, Munhwan KIM \\ Department of Surgery, Seoul National University Bundang Hospital, Seongnam, Korea
}

Introduction: The safety of laparoscopic liver resection (LLR) in elderly patients is a matter of concern because reduced physiologic reserve increased the risk of morbidity. However, few studies have been designed to develop scoring system to predict complications after LLR in elderly patients. This study aimed to investigate the clinical value of the geriatric nutritional risk index (GNRI) and propose a new simplified scoring system for predicting the risk of postoperative major complications after LLR in elderly hepatocellular carcinoma (HCC) patients.

Methods: We retrospectively reviewed 257 consecutive patients aged $\geq 65$ years who underwent LLR for HCC from January 2004 to December 2019. The GNRI formula was calculated as follows: $1.489 \mathrm{x}$ serum albumin $(\mathrm{g} / \mathrm{L})+41.7 \mathrm{x}$ present weight/ideal weight $(\mathrm{kg})$.

Results: Major complication following LLR was noted in 24 patients (10.9\%). On multivariate analysis, the GNRI (hazard ratio [HR] 3.396, 95\% confidence interval [CI] 1.242-9.288, $p=0.017$ ), Child-Pugh score (HR 2.191, 95\% CI 1.400-8.999, $p=0.036$ ), major liver resection (HR 2.683, 95\% CI 1.082-7.328) and intraoperative transfusion (HR 1.802, 95\% CI 1.428-7.591, $p=0.022$ ) independently predicted postoperative major complications. Four significant predictors were identified and assigned points based on hazard ratio. The 10 -point model showed good discrimination (Area under the curve 0.756, 95\% CI 0.649-0.836, $p=0.001$ ).

Conclusions: The GNRI is an important predictor of postoperative major complications and the proposed risk score can help to predict the risk of planned surgery in elderly HCC patients. 